\title{
Observation of Inverse Diproton Photodisintegration at Intermediate Energies
}

\author{
V. Komarov, ${ }^{1}$ T. Azaryan, ${ }^{1}$ D. Chiladze, ${ }^{2,3}$ S. Dymov, ${ }^{1,4, *}$ A. Dzyuba, ${ }^{6}$ M. Hartmann, ${ }^{2}$ A. Kacharava, ${ }^{2}$ I. Keshelashvili, ${ }^{2,3}$ \\ A. Khoukaz, ${ }^{5}$ A. Kulikov, ${ }^{1}$ V. Kurbatov, ${ }^{1}$ G. Macharashvili, ${ }^{1,3}$ S. Merzliakov, ${ }^{1,2}$ S. Mikirtychiants, ${ }^{2,6}$ M. Papenbrock, ${ }^{5}$ \\ M. Nekipelov, ${ }^{2,6}$ F. Rathmann, ${ }^{2}$ V. Serdyuk, ${ }^{1,2}$ H. Ströher, ${ }^{2}$ D. Tsirkov, ${ }^{1}$ Yu. Uzikov, ${ }^{1}$ and C. Wilkin ${ }^{7}$ \\ ${ }^{1}$ Laboratory of Nuclear Problems, Joint Institute for Nuclear Research, 141980 Dubna, Russia \\ ${ }^{2}$ Institut für Kernphysik, Forschungszentrum Jülich, 52425 Jülich, Germany \\ ${ }^{3}$ High Energy Physics Institute, Tbilisi State University, 0186 Tbilisi, Georgia \\ ${ }^{4}$ Physikalisches Institut II, Universität Erlangen-Nürnberg, 91058 Erlangen, Germany \\ ${ }^{5}$ Institut für Kernphysik, Universität Münster, 48149 Münster, Germany \\ ${ }^{6}$ High Energy Physics Department, Petersburg Nuclear Physics Institute, 188350 Gatchina, Russia \\ ${ }^{7}$ Physics and Astronomy Department, UCL, London, WCIE 6BT, United Kingdom \\ (Received 3 June 2008; revised manuscript received 23 July 2008; published 3 September 2008)
}

The fundamental reaction $p p \rightarrow\{p p\}_{s} \gamma$, where $\{p p\}_{s}$ is a proton pair with low excitation energy, has been observed with the ANKE spectrometer at COSY-Jülich for proton beam energies of $T_{p}=0.353$, 0.500 , and $0.550 \mathrm{GeV}$. This is equivalent to photodisintegration of a free ${ }^{1} S_{0}$ diproton for photon energies $E_{\gamma} \approx T_{p} / 2$. The differential cross sections measured for c.m. angles $0^{\circ}<\theta_{p p}<20^{\circ}$ exhibit a steep increase with angle that is compatible with $E 1$ and $E 2$ multipole contributions. The ratio of the measured cross sections to those of $n p \rightarrow \gamma d$ is on the $10^{-3}-10^{-2}$ level. The increase of the $p p \rightarrow\{p p\}_{s} \gamma$ cross section with $T_{p}$ might reflect the influence of the $\Delta(1232)$ excitation.

DOI: 10.1103/PhysRevLett.101.102501

Photoabsorption on two-nucleon systems in nuclei at several hundred $\mathrm{MeV}$ allows one to probe fundamental properties of nuclei at short distances. The photodisintegration of the simplest nucleus, the deuteron, through the $\gamma d \rightarrow p n$ reaction is widely used as a testing ground for different theoretical ideas of the nucleon-nucleon interaction, such as meson-exchange models and isobar currents [1] or, more recently, quark-gluon degrees of freedom [2]. However, much less is known, both experimentally and theoretically, on the other simplest process

$$
\gamma+\{p p\}_{s} \rightarrow p+p
$$

where $\{p p\}_{s}$ is a proton pair in the ${ }^{1} S_{0}$ state. The photodisintegration of the spin-singlet $p p$-pair differs from that of the spin-triplet $\left({ }^{3} S_{1}-{ }^{3} D_{1}\right)$ pn pair, where the $M 1$ magnetic dipole transition dominates $\gamma d \rightarrow p n$ at several hundred $\mathrm{MeV}$ through the excitation of the $\Delta(1232)$ isobar $[3,4]$. In contrast, due to selection rules, there is no direct contribution to reaction (1) from $S$-wave $\Delta N$ intermediate states [5,6] and $M$-odd multipoles are forbidden. Furthermore, since the diproton has no electric dipole moment, only the spin-flip contribution to the $E 1$ operator survives [6]. Features of the underlying dynamics, which are not visible in the photodisintegration of the deuteron, may therefore reveal themselves in reaction (1).

In the absence of a free bound diproton, reaction (1) has only been investigated for a ${ }^{1} S_{0}$ diproton bound within a nucleus, the lightest of these being ${ }^{3} \mathrm{He}$ [7-9]. However, since the $M 1$ absorption on quasideuteron pairs is so strong, the ${ }^{3} \mathrm{He}(\gamma, p p) n$ reaction has large backgrounds associated with apparent three-nucleon absorption, combined with final state interactions (FSI). The total cross
PACS numbers: 25.40.Ep, 13.60.-r, 25.20.Dc

section for photon absorption by two protons in ${ }^{3} \mathrm{He}$ for photon energies $0.2-0.5 \mathrm{GeV}$ was found to be only a few percent of the total rate [7]. These contaminations are absent in the inverse reaction with the production of a free ${ }^{1} S_{0}$ diproton

$$
p+p \rightarrow \gamma+\{p p\}_{s} .
$$

At excitation energies $E_{p p}$ of the final $p p$ pair less than a few $\mathrm{MeV}$ ( $E_{p p}<3 \mathrm{MeV}$, for definiteness), the system is almost exclusively in the ${ }^{1} S_{0}$ state.

The known experiments on hard $p p$ bremsstrahlung at intermediate energies were not designed for the study of the quasi-two-body channel (2). In the published data [1015], the selection of low $E_{p p}$ events was either impossible instrumentally or was not done if feasible. In the COSYTOF experiment at a beam energy of $T_{p}=0.293 \mathrm{GeV}$, the $p p \gamma$ data did not exhibit any sizable FSI enhancement at low $E_{p p}$ and no estimate of the cross section for channel (2) was made [16]. The aim of the present work was to identify the fundamental reaction (2) in the region of the $\Delta(1232)$ and to investigate its differential cross section. Here we present the first results of measurements at $T_{p}=0.353$, 0.500 , and $0.550 \mathrm{GeV}$.

The experiment was performed at the ANKE facility [17] of the synchrotron storage ring COSY-Jülich. The acceptance of the system is shown in Fig. 1(a). It is seen that the setup allows the recording of protons from reaction (2) for $\left|\theta_{X Z}\right| \lesssim 5^{\circ}$. The vertical acceptance is $\pm 3.5^{\circ}$. The acceptance for the proton pairs peaks at small polar angles $\theta_{p p}$ [inset in Fig. 1(a)].

Single protons from elastic $p p$ scattering were used, together with the differential cross sections from Ref. [18], to 

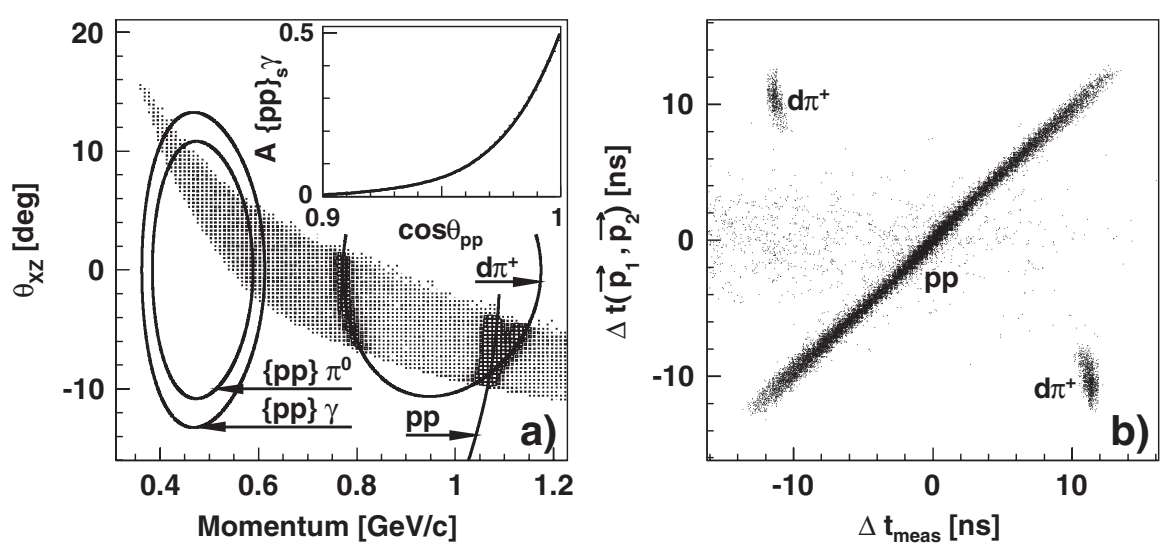

FIG. 1. Performance of the setup at 0.5 $\mathrm{GeV}$. (a) The polar angle projection $\theta_{X Z}$ onto the ANKE median plane plotted versus the momentum of single particles. The curves depict kinematical loci for $p$ and $d$ from the indicated processes. Here $\{p p\}$ denotes a $p p$ pair with $E_{p p}=0$. The acceptance $A\{p p\}_{s} \gamma$ for reaction (2) is shown in terms of the c.m. polar angle $\theta_{p p}$ of the proton pair. (b) Identification of the proton pairs, as described in the text. establish the absolute normalization. The errors in the integrated luminosities in Table I include both systematic and normalization effects. Detailed descriptions of the setup and data processing are given in Refs. [19-21].

When proton pairs hit different counters, the difference $\Delta t_{\text {meas }}$ of the arrival times can be measured and compared with the time-of-flight difference $\Delta t\left(\vec{p}_{1}, \vec{p}_{2}\right)$ calculated using the measured momenta, assuming that both particles are protons. The $\Delta t_{\text {meas }}-\Delta t\left(\vec{p}_{1}, \vec{p}_{2}\right)$ distribution has a FWHM of $0.6-1.1 \mathrm{~ns}$, so that genuine proton coincidences can be identified unambiguously [Fig. 1(b)]. The tracking system led to a precision in the determination of the momentum $\sigma(p) / p \approx 1 \%$ and polar angle $\sigma(\theta) \approx 0.2^{\circ}$ for protons around $0.6 \mathrm{GeV} / c$ and these gave a resolution $\sigma\left(E_{p p}\right)=0.1-0.5 \mathrm{MeV}$ for $E_{p p}<3 \mathrm{MeV}$.

The determination of the four momenta of the two final protons allows a full kinematical reconstruction of $p p \rightarrow$ $p p X$ events and the derivation of the missing-mass spectra for the pairs with $E_{p p}<3 \mathrm{MeV}$. In our previous work at $T_{p}=0.625$ and $0.8 \mathrm{GeV}[20,21]$, only a hint of reaction (2) could be seen. For the present energies, a clear peak is revealed around $M_{x}^{2} \approx 0$ (Fig. 2). This is well separated from the $\pi^{0}$ signal at $0.353 \mathrm{GeV}$ whereas, at 0.5 and $0.55 \mathrm{GeV}$, the two structures partially overlap because of broadening of the pion peak away from the production threshold. Fits of the $M_{x}^{2}$ distributions as the sum of modeled $\gamma$ and $\pi^{0}$ contributions and a straight line background lead to the parameters listed in Table I. The missing-energy distributions for the $\gamma$-peak events in the overall c.m. frame, which are reflections of the resolution of the setup and the $E_{p p}$ range, have widths $\approx 1 \mathrm{MeV}$. The mean $E_{x}^{m}$ in Table I agree with the expected kinematic values to within $\approx 0.2 \mathrm{MeV}$. The energy $E_{\gamma}$ of the inverse reaction is averaged over the $E_{p p}$ range $0-3 \mathrm{MeV}$ and distributed with an rms of $0.5 \mathrm{MeV}$.

The $E_{p p}$ spectrum of events from the $\gamma$ peak is consistent with pure ${ }^{1} S_{0}$ production. Simulated events, generated according to phase space and modified by a $p p$ FSI factor taken from the square of the low-energy $S$-wave $p p$ elastic amplitude [20], were traced through the experimental setup. The simulation satisfactorily reproduces the experiment, with $\chi^{2} / \mathrm{ndf}=11.4 / 11$ at $0.5 \mathrm{GeV}$. If the FSI is neglected, this figure rises to $71 / 11$. Further evidence that $P$-wave contamination is small is provided by the acceptance-corrected proton angular distribution in the $p p$ rest frame, which is consistent with isotropy.

To obtain the differential cross section $d \sigma / d \Omega_{p p}$ as a function of the diproton polar angle $\theta_{p p}$, events with $E_{p p}<3 \mathrm{MeV}$ in the $\gamma$ peak of the $M_{x}^{2}$ distributions were analyzed in $\cos \theta_{p p}$ bins of $0.01-0.02$ width. After subtraction of the background contamination, the yield of reaction (2) was found from the number of events in the intervals $M_{x}^{2}=0 \pm 2.5 \sigma\left(M_{x}^{2}\right)_{\gamma}$ at $0.353 \mathrm{GeV}$ and $M_{x}^{2}=0 \pm$ $1.8 \sigma\left(M_{x}^{2}\right)_{\gamma}$ at higher energies. The background was determined at $0.353 \mathrm{GeV}$ by using $M_{x}^{2}$ intervals outside but adjacent to the $\gamma$ peak. The contribution from the tail of the $\pi^{0}$ peak was more important at 0.5 and $0.55 \mathrm{GeV}$. The $\pi^{0}$ shape was taken from the simulation and the quality of the description of the tails was controlled by the data on the high mass side of the peak.

The differential cross section is a function of $x=$ $\cos ^{2} \theta_{p p}$, and the results given in Table II indicate a very strong dependence upon this variable. Theoretical considerations of the $\gamma\{p p\}_{s} \rightarrow p p$ reaction [22] suggest that, in

TABLE I. Measurement characteristics: $L_{\text {int }}$ is the integrated luminosity; $\left(M_{x}^{2}\right)^{m}$ and $\sigma\left(M_{x}^{2}\right)$ in $0.01 \mathrm{GeV}^{2} / c^{4}$ units are, respectively, the mean value and the standard deviation of the missing-mass-squared distributions for $p p$ pairs with $E_{p p}<$ $3 \mathrm{MeV}$ at the beam energy $T_{p} ; N_{\gamma}$ is the number of events in the $\gamma$ peak for $\theta_{p p}<20^{\circ} ; N_{\mathrm{bg}} / N_{\gamma}$ is the ratio of the background to signal in the $\gamma$ peak; $E_{x}^{m}$ is the mean value of the missing c.m. energy for the events from the $\gamma$ peak; $E_{\gamma}$ is the laboratory energy of the photon in the inverse reaction (1).

\begin{tabular}{lccc}
\hline \hline$T_{p}[\mathrm{GeV}]$ & 0.353 & 0.500 & 0.550 \\
$E_{\gamma}[\mathrm{GeV}]$ & 0.176 & 0.249 & 0.274 \\
\hline$L_{\mathrm{int}}\left[\mathrm{nb}^{-1}\right]$ & $573 \pm 18$ & $331 \pm 10$ & $318 \pm 21$ \\
$\left(M_{x}^{2}\right)_{\gamma}^{m}$ & $0.01 \pm 0.03$ & $0.02 \pm 0.04$ & $0.01 \pm 0.04$ \\
$\sigma\left(M_{x}^{2}\right)_{\gamma}$ & $0.28 \pm 0.04$ & $0.35 \pm 0.03$ & $0.41 \pm 0.02$ \\
$N_{\gamma}$ & 180 & 335 & 525 \\
$N_{\mathrm{bg}} / N_{\gamma}$ & $0.23 \pm 0.04$ & $0.05 \pm 0.03$ & $0.11 \pm 0.07$ \\
$E_{x}^{m}[\mathrm{GeV}]$ & 0.161 & 0.221 & 0.241 \\
\hline \hline
\end{tabular}




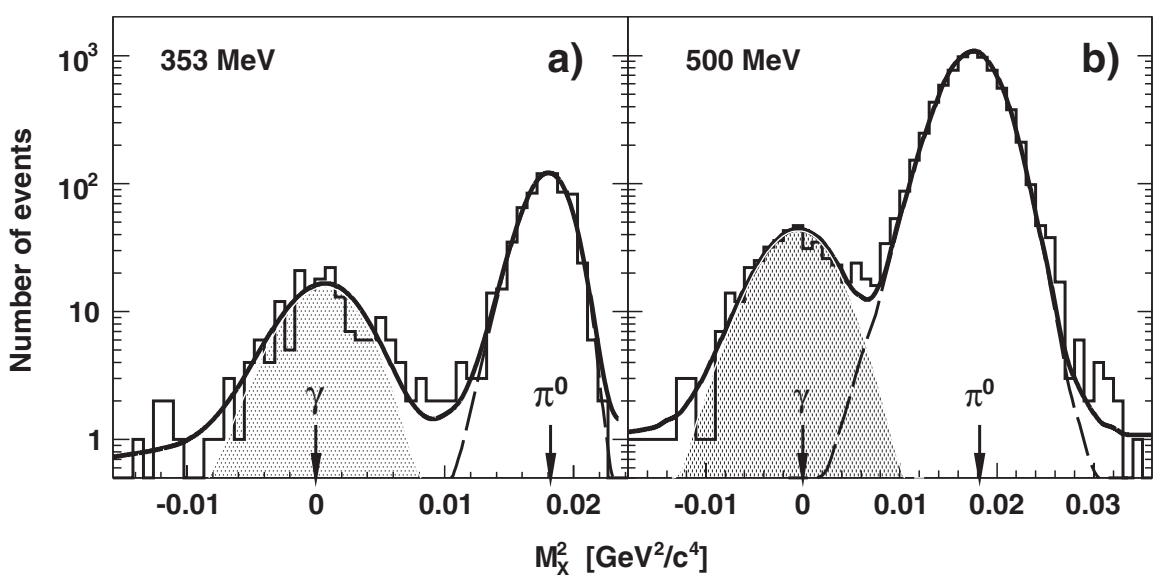

FIG. 2. Missing-mass-squared distributions for the $p p \rightarrow p p X$ reaction for events with $E_{p p}<3 \mathrm{MeV}$. The shaded area corresponds to the predicted $\gamma$ peak, the dashed line to the $\pi^{0}$, and the solid to the sum of these and a straight line background.

our energy range, it might be sufficient to retain transitions corresponding to only the three lowest multipoles, viz. E1, $E 2$, and $M 2$. Moreover, it is predicted [6] that the $M 2$ strength should vanish for $E_{\gamma} \approx 0.25 \mathrm{GeV}$ and be rather low compared to $E 1$ and $E 2$ in the range $0.18-0.28 \mathrm{GeV}$. Since the $E 2$ and $E 1$ transitions do not interfere, restricting to just these two multipoles, the differential cross section is of the form

$$
\frac{d \sigma}{d \Omega_{p p}}=a[(1+x) \kappa+10 x(1-x)],
$$

where $\kappa=\sigma(E 1) / \sigma(E 2)$ and $a=3 \sigma(E 2) / 16 \pi$. Here $\sigma(E J)$ is the total cross section of reaction (2) for the $E J$ multipole. Fitting the data with this form [see Fig. 3(a)] leads to the parameters $\kappa$ and $a$ given in Table II. For all our energies the value of $\kappa$ shows that the $E 1$ and $E 2$ multipoles have rather similar strengths, a feature that was not evident in the ${ }^{3} \mathrm{He}(\gamma, p p) n$ experiments [7-9].

The cross sections for the $p p \rightarrow\{p p\}_{s} \gamma$ reaction are compared in Fig. 3(b) with those of $n p \rightarrow d \gamma$ [3]. The diproton-to-deuteron ratio is small and varies with angle and energy between about $4 \times 10^{-3}$ and $3 \times 10^{-2}$. In part, this low value is due to the smaller phase space volume for the unbound $p p$ system and this gives a suppression factor $\approx 0.1$ [20]. The residual suppression must be related to the different dynamics in the two reactions. The crucial point here is the absence in the diproton photodisintegration of the spin-non-flip E1 term [6] and the $M 1$ transition [5], which dominates the $\gamma d \rightarrow p n$ reaction in the $\Delta$ energy range. Intermediate $\Delta N$ states are allowed in $P$ and higher partial waves [6], though their strength will be reduced by the centrifugal barrier. As a consequence, the contribution of the $\Delta$ isobar in the $\gamma\{p p\}_{s} \rightarrow p p$ absorption should be greatly diminished compared with the $\gamma d \rightarrow p n$ case. This logic has also been advanced to explain the relatively small rate of diproton photodisintegration in ${ }^{3} \mathrm{He}(\gamma, p p) n$ [7-9].

The most prominent feature in the energy dependence of the $\gamma d \rightarrow p n$ total and small-angle differential cross sections is the bump at $E_{\gamma} \approx 150-300 \mathrm{MeV}$ [3], caused by the excitation of the $\Delta$ isobar. In contrast, the total cross section for photon absorption by two bound protons in the ${ }^{3} \mathrm{He}(\gamma, p p) n$ reaction [7] falls steadily as $E_{\gamma}$ increases from 0.2 to $0.5 \mathrm{GeV}$, in qualitative agreement with the arguments for the $\Delta$-isobar suppression. It is also in line with the results of the model calculation that indicates a monotonic decrease in the $E 2$ contribution through the $\Delta$ region [6]. Our results are in clear disagreement with these findings. It is important to note that, if the $M 2$ amplitude is neglected, the parameter $a$ would reflect directly the $E 2$ contribution to the $p p \rightarrow\{p p\}_{s} \gamma$ total cross section. The values of $a$ reported in Table II increase strongly with energy and the most plausible explanation for this behavior

TABLE II. Differential cross sections of the $p p \rightarrow\{p p\}_{s} \gamma$ reaction in the c.m. system. The errors shown take into account only statistics and uncertainties in the background estimation. The overall normalization is known to about $\pm 11 \%$. The fit parameters $\kappa$ and $a$ are defined by Eq. (3).

\begin{tabular}{|c|c|c|c|c|c|c|}
\hline \multirow[t]{2}{*}{$T_{p}[\mathrm{GeV}]$} & \multicolumn{2}{|c|}{0.353} & \multicolumn{2}{|c|}{0.500} & \multicolumn{2}{|c|}{0.550} \\
\hline & $\begin{array}{c}\theta_{p p} \\
{[\mathrm{deg}]}\end{array}$ & $\begin{array}{c}d \sigma / d \Omega_{p p} \\
{[\mathrm{nb} / \mathrm{sr}]}\end{array}$ & $\begin{array}{c}\theta_{p p} \\
{[\mathrm{deg}]}\end{array}$ & $\begin{array}{c}d \sigma / d \Omega_{p p} \\
{[\mathrm{nb} / \mathrm{sr}]}\end{array}$ & $\begin{array}{c}\theta_{p p} \\
{[\mathrm{deg}]}\end{array}$ & $\begin{array}{c}d \sigma / d \Omega_{p p} \\
\quad[\mathrm{nb} / \mathrm{sr}]\end{array}$ \\
\hline & 5.6 & $3.7 \pm 0.8$ & 4.8 & $10.4 \pm 1.5$ & 4.8 & $20.4 \pm 2.6$ \\
\hline & 10.2 & $4.3 \pm 0.9$ & 8.1 & $16.1 \pm 3.0$ & 8.1 & $22.1 \pm 3.2$ \\
\hline & 13.6 & $6.8 \pm 1.3$ & 11.0 & $14.7 \pm 2.4$ & 11.0 & $27.8 \pm 3.4$ \\
\hline & 17.7 & $5.4 \pm 1.1$ & 14.1 & $17.2 \pm 2.9$ & 14.1 & $34.5 \pm 5.3$ \\
\hline & & & 17.9 & $20.7 \pm 3.5$ & 17.9 & $35.7 \pm 5.7$ \\
\hline$a[\mathrm{nb} / \mathrm{sr}]$ & \multicolumn{2}{|c|}{$\begin{array}{c}3.1 \pm 1.7 \\
0.58+0.44\end{array}$} & \multicolumn{2}{|c|}{$13.5 \pm 4.1$} & \multicolumn{2}{|c|}{$23.8 \pm 6.9$} \\
\hline$\kappa$ & \multicolumn{2}{|c|}{$0.58 \pm 0.44$} & \multicolumn{2}{|c|}{$0.38 \pm 0.16$} & \multicolumn{2}{|c|}{$0.40 \pm 0.16$} \\
\hline
\end{tabular}




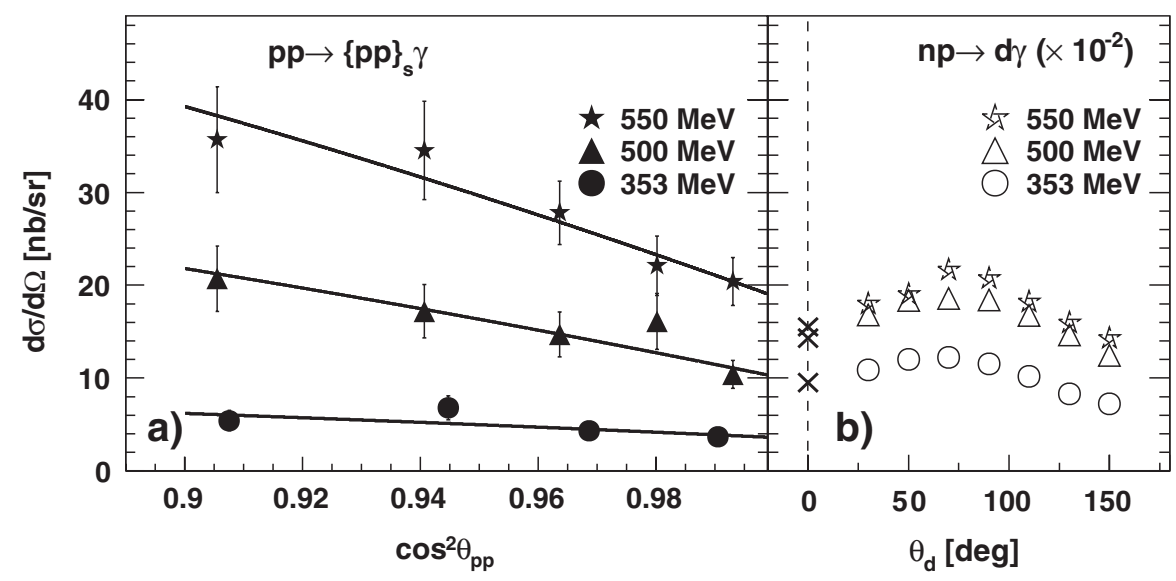

FIG. 3. (a) Angular dependence of the c.m. differential cross section of the $p p \rightarrow\{p p\}_{s} \gamma \quad$ reaction. The errors shown do not include that of the absolute normalization. The full curves represent the fits on the basis of Eq. (3), with the parameters being given in Table II. (b) Differential cross section for the $n p \rightarrow d \gamma$ reaction deduced from data on the inverse reaction [3]. Crosses at $\theta_{d}=0^{\circ}$ are theoretical expectations [4] for energies (down to up) of 353, 500, $550 \mathrm{MeV}$. is the influence of $D$-wave $\Delta N$ intermediate states. A ${ }^{5} S_{2}$-wave $\Delta N$ component, excited indirectly in the $E 2$ transition [6], may also be a reason for this behavior.

A similar rapid rise with angle was noted in the differential cross section for single pion production in the $p p \rightarrow$ $\{p p\}_{s} \pi^{0}$ reaction near the forward direction [21]. The $S$-wave $\Delta N$ contribution is also suppressed here by parity and angular momentum conservation, though a broad maximum was observed in the forward direction at 0.5$1.0 \mathrm{GeV}$, which might be a reflection of higher partial waves in the $\Delta N$ intermediate states.

In a further investigation of the $p p \rightarrow\{p p\}_{s} \gamma$ reaction at ANKE, the analyzing power will be measured, together with that for $p p \rightarrow\{p p\}_{s} \pi^{0}$, over an extended angular range using a polarized proton beam [23]. A signal should arise from the interference of the $E 2$ with the $E 1$ and $M 2$ multipoles [22].

An extended study of reaction (2), involving also the use of $\gamma$ detectors, might be feasible at COSY, where the maximum beam energy is $T_{p}=2.9 \mathrm{GeV}$. An investigation at energies well above the $\Delta$ region would allow one to compare with other ${ }^{3} \operatorname{He}(\gamma, p p) n$ data [8]. The onset of dimensional scaling, observed at large transverse momenta in $\gamma d \rightarrow p n$ for $E_{\gamma}>1 \mathrm{GeV}$ [24] and suggested for ${ }^{3} \mathrm{He}(\gamma, p p) n$ [25], might also be studied in reaction (2).

In summary, the reaction $p p \rightarrow\{p p\}_{s} \gamma$ with production of the final ${ }^{1} S_{0}$ proton pair has been observed at intermediate energies. The feasibility of measuring the essential characteristics of the reaction has been demonstrated. The absolute values of the small-angle cross section are orders of magnitude lower than for $n p \rightarrow d \gamma$ and its unexpected rise with energy may be related to $\Delta(1232)$ excitation. The rapid variation of the cross section with angle allows an estimate to be made of the ratio of the $E 1$ and $E 2$ multipole intensities. Crucially, the reaction does not suffer from the drawbacks inherent in photoabsorption on ${ }^{3} \mathrm{He}$, i.e., the presence of multinucleon absorption. A detailed study of this fundamental reaction might open up a new way to investigate the properties of the $p p$ system at high momentum transfers under conditions that allow a clean interpretation.

Discussions with S. B. Gerasimov and K. Nakayama were helpful. The work was partially financed by the BMBF Grant to JINR and supported by COSY FFE and HGF VIQCD.

*s.dymov@fz-juelich.de

[1] H. Arenhövel and M. Sanzone, Few-Body Syst. Suppl. 3, 1 (1991).

[2] R. Gilman and F. Gross, J. Phys. G 28, R37 (2002).

[3] R. Crawford et al., Nucl. Phys. A603, 303 (1996).

[4] P. Wilhelm and H. Arenhövel, Phys. Lett. B 318, 410 (1993).

[5] J. M. Laget, Nucl. Phys. A497, 391c (1989).

[6] P. Wilhelm, J. A. Niskanen, and H. Arenhövel, Phys. Rev. C 51, 2841 (1995).

[7] T. Emura et al., Phys. Rev. Lett. 73, 404 (1994).

[8] S. Niccolai et al., Phys. Rev. C 70, 064003 (2004).

[9] G. Audit et al., Phys. Lett. B 312, 57 (1993).

[10] B. M. K. Nefkens et al., Phys. Rev. C 19, 877 (1979).

[11] K. Yasuda et al., Phys. Rev. Lett. 82, 4775 (1999).

[12] K. Michaelian et al., Phys. Rev. D 41, 2689 (1990).

[13] B. V. Przewoski et al., Phys. Rev. C 45, 2001 (1992).

[14] J. Złomańczuk and A. Johansson, Nucl. Phys. A631, 622 (1998).

[15] M. Mahjour-Shafiei et al., Phys. Rev. C 70, 024004 (2004).

[16] R. Bilger et al., Phys. Lett. B 429, 195 (1998).

[17] S. Barsov et al., Nucl. Instrum. Methods Phys. Res., Sect. A 462, 364 (2001).

[18] R. A. Arndt et al., Phys. Rev. C 76, 025209 (2007); http:// gwdac.phys.gwu.edu, SAID solution S P07.

[19] S. Dymov et al., Part. Nucl. Lett. 1, 40 (2004).

[20] S. Dymov et al., Phys. Lett. B 635, 270 (2006).

[21] V. Kurbatov et al., Phys. Lett. B 661, 22 (2008).

[22] P. Wilhelm, J. A. Niskanen, and H. Arenhövel, Nucl. Phys. A597, 613 (1996).

[23] A. Kulikov et al., COSY proposal \#158, http://www.fzjuelich.de/ikp/anke/en/proposals.shtml.

[24] P. Rossi et al., Phys. Rev. Lett. 94, 012301 (2005).

[25] S. J. Brodsky et al., Phys. Lett. B 578, 69 (2004). 